\title{
Pneumothorax as a Complication of Radiation Therapy for Primary Lung Cancer
}

\author{
Michael R. HAY, M.D. \\ Kyung J. Cho, M.D. \\ Hyunchul Shin, M.D.
}

From the Departments of Radiology, University of Michigan Medical Center, Ann Arbor, Michigan 48109 and Wayne County General Hospital, Eloise, Michigan.

\section{CASE REPORT}

A 57-year-old man was evaluated at another hospital for weakness, weight loss, and shortness of breath, where bronchial biopsy of a mass in the right main stem bronchus was negative but washings showed squamous cell carcinoma. He was then referred to Wayne County General Hospital. Chest x-ray and xerotomograms showed opacification of the right hemithorax with some shift of the mediastinum to the right and amputation of the right main bronchus (Figure 1). Bronchoscopy showed a mass obstructing the right main stem bronchus, extending into the trachea. Biopsy revealed poorly differentiated bronchogenic car-

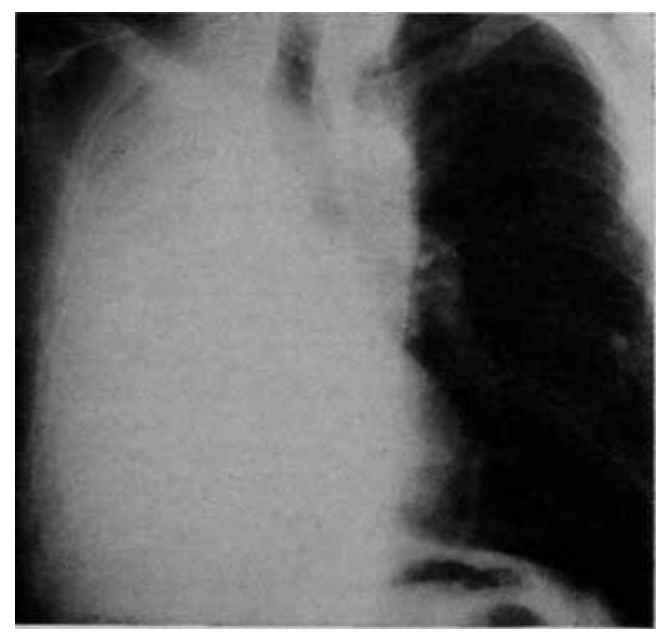

FIGURE 1A-Frontal view of the chest before irradiation. There is opacification of right hemithorax with shift of the mediastinum and deviation of the trachea to the right due to a collapse of right lung. cinoma, probably squamous type. Radiation therapy with cobalt was begun on 10-15-75 and ended $11-10-75$ at which time 4000 rads had been given to the mediastinum and right hilum. During the earlier course of radiotherapy he developed acute facial cyanosis and distension of neck veins. Superior vena cavography revealed complete occlusion of the superior vena

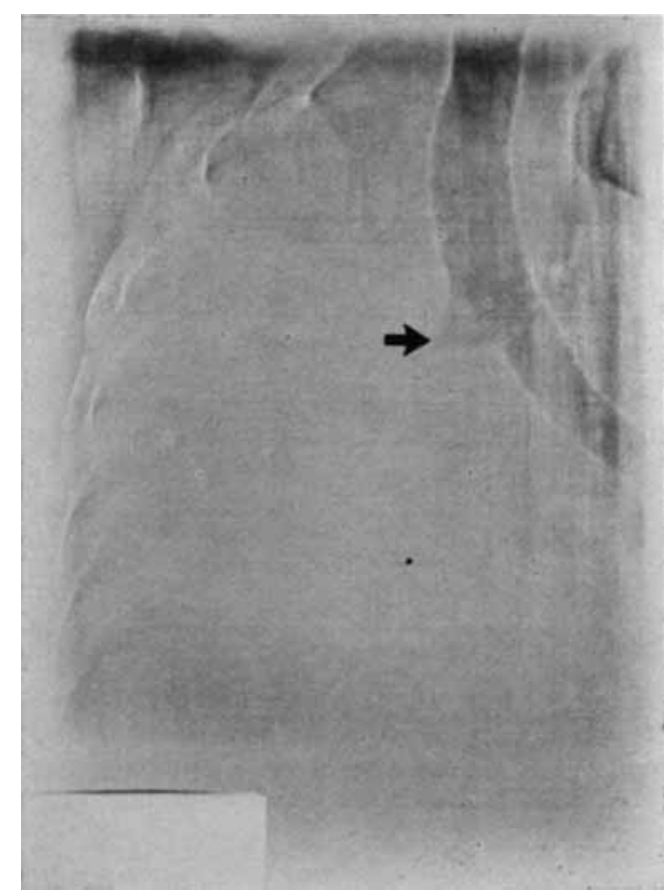

FIGURE 1B-Xerotomogram of tracheobronchial tree. Right main bronchus is amputated with a tapering appearance characteristic of bronchogenic carcinoma (arrow).

Australasian Radiology, Vol. XX, No. 4, December, 1976 


\section{Pneumothorax as a Complication of Radiation Therapy for Primary lung Cancer}

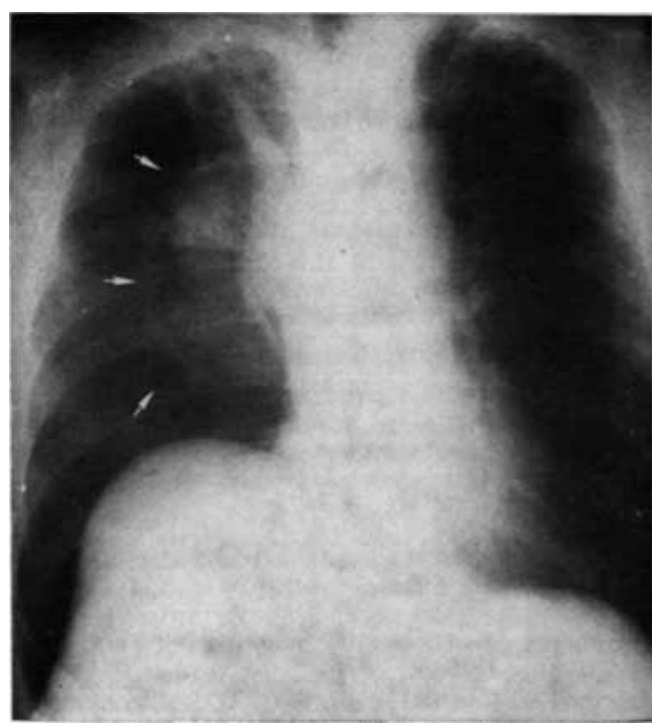

FIGURE 2-Chest x-ray after completion of irradiation. There is massive pneumothorax with a collapsed right lung (arrows).

cava. Later during his radiation therapy signs and symptoms of the superior vena cava syndrome markedly regressed. Because of increasing shortness of breath and chest discomfort after completion of radiotherapy, a chest roentgenogram was obtained and showed a massive pneumothorax in a previously opacified hemithorax (Figure 2). The patient then died. Autopsy was not granted.

\section{Discussion}

There have been many reports of cases of pneumothorax associated with lung tumours, but most of these have been of metastatic tumours", particularly osteogenic sarcomas. Spontaneous pneumothorax has also been reported to $b_{2}$ a complication of bronchogenic carcinoma, but extremely rare $^{1}$. Most of these cases in the literature have either presented with pneumothorax or have occurred during diagnostic work-up, not including iatrogenic cases. The patients presented by Dines et al. ${ }^{3}$, had pulmonary metastases at the time of pneumothorax, which could have increased the likelihood of pneumothorax. It has been emphasised that spontaneous pneumothorax could be a clue to an unsuspected bronchogenic carcinoma ${ }^{10}$.

Libshitz $^{8}$ described two cases of pneumothorax following cobalt irradiation of the chest for Hodgkin's disease occurring 8 months and 4 months post treatment and theorised them to be related to radiation induced fibrosis and emphysematous change with ruptured subpleural blebs.

Citron $^{2}$ presented six cases in 1959, five of which were proven cases of bronchogenic carcinoma and in which the patients either presented with the pneumothorax or developed it during the course of the illness, apparently prior to treatment. His sixth case was different in that bronchoscopy showed narrowing of the left lower lobe bronchus but histologic diagnosis was not made. Radiotherapy was given and a left pneumothorax developed "soon after." Bayden et al. ${ }^{1}$, reported a case of squamous cell carcinoma of the right main bronchus where a right pneumothorax and pneumopericardium developed 6 days after cobalt treatment was begun. The patient died several weeks later; no autopsy was performed.

Several mechanisms have bzen shown or postulated to explain the association of bronchogenic carcinoma with pneumothorax. One is that the association is fortuitous since bronchogenic carcinoma and spontaneous pneumothorax are common entities. The occurrence on the same side is harder to explain. Another explanation is the creation of a bronchopleural fistula by a tumour invading the pleura which becomes necrotic. This has been demonstrated in other reports ${ }^{5.9}$. A third mechanism is that a partially obstructing tumour produces obstructive emphysema and bleb formation causing a pneumothorax when the bleb ruptures ${ }^{3.6 .9}$.

In the present case, pneumothorax was closely related temporally to the radiation therapy. Apparently the patient's tumour responded to the treatment as evidenced by remission of the superior vena cava syndrome. $A$ reasonable inference could be that the tumour became necrotic resulting in a bronchopleural fistula.

\section{Abstract}

A case of spontaneous pneumothorax complicating irradiation for bronchogenic carcinoma is presented. Pneumothorax developed in a collapsed lung caused by a central bronchogenic carcinoma. The cause is presumably secondary to either a bronchopleural fistula by tumour or sudden expansion of the lung following irradiation.

Various tumours of the lung have been associated with spontaneous pneumothorax, 


\section{Michael R. Hay, Kyung J. Cho and Hyunchul Shin}

including metastatic osteogenic sarcoma ${ }^{12}$, eosinophilic granuloma ${ }^{4}$, teratoma ${ }^{11}$, other metastatic sarcomas ${ }^{3}$, lymphoma following radiation therapy ${ }^{*}$, and primary bronchogenic carcinomas $1,2,3,3,1,7,9,10$,

We have encountered a case of spontaneous pneumothorax complicating irradiation for bronchogenic carcinoma. To our knowledge, this is the first report in the English radiologic literature, and the second, in the English literature, of a case of spontaneous pneumothorax following radiation therapy for proven bronchogenic carcinoma.

\section{REFERENCES}

'Bayden, A., and Gottleib, L. (1976): "Pneumopericarcium and Pneumothorax Complicating Bronchogenic Carcinoma." Western J. Med., 124, 144.

${ }^{2}$ Citron, K. M. (1959): "Spontaneous Pneumothorax Complicating Bronchial Carcinoma." Tubercle, 40, 384 .

${ }^{3}$ Dines, D, E., Cortese, D.. Brennan, M., Hahn, R., Payne, W. (1973): "Malignant Pulmonary Neoplasms Predisposing to Spontaneous Pneumothorax." Mayo Clin. Proc., 48, 541.

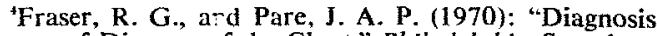
of Diseases of the Chest." Philadelphia, Saunders, 1116.

'Gautam, H. P., and Knight, E. V. (1970): "Spontaneous Preumothorax Due to Perforated Bronchial Carcinoma." Brit. J. Dis. Chest, 64, 110.

"Heimlich, H. J., and Ruben, M. (1955): "Spontaneous Pneumothorax as a Prese -ting Feature of Primary Carcinoma of the Lung." Dis. Chest, 27, 457.

'Khan, F., and Seriff, N. (1973): "Pneumothorax. A Rare Presenting Manifestation of Lung Cancer." Ann. Rer. Resp. Dis., 108, 1397.

${ }^{\mathrm{x}}$ Libshitz, H., and Banner, M. (1974): "Spontaneous Preumothorax as a Complication of Radiation Therapy to the Thorax." Radiology, 112, 119.

'Rose, M. E., Howard, R., Silbey, J. J., and Reilly, H. F. Jr. (1967): "Pneumothorax Directly Resulting from Perforated Bronchogenic Carcinoma." Ann. Thorac. Surg., 4, 160.

${ }^{10}$ Seremetis, M. G., and MacDonald, J. C. (1970): "Spontaneous Pneumothorax and Carcinoma of Lung. A Case Report." Milit. Med., 135, 898.

${ }^{11}$ Singh, A., Sethi, R., and Singh, G. (1973): "Pneumothorax: An Unusual Complication of Teratoma." Chest, 63, 1034.

${ }^{12}$ Swaney, J. J., and Cangir, A. (1973): "Spontaneous Pneumothorax in Metastatic Osteogenic Sarcoma." J. Pediatr., 82, 165. 